\title{
XML Query Optimization in the Presence of Side Effects
}

\begin{abstract}
The emergence of database languages with side effects, notably for XML, raises significant challenges for database compilers and optimizers. In this paper, we extend an algebra for the W3C XML query language with operations that allow data to be immediately updated. We study the impact of that extension on logical optimization, join detection, and pipelining. The main result of this work is to show that, with proper care, a number of important optimizations based on nested relational algebras remain applicable in the presence of side effects. Our approach relies on an analysis of the conditions that must be checked in order for algebraic rewritings to hold. An implementation and experimental results demonstrate the effectiveness of the approach.
\end{abstract}

\section{INTRODUCTION}

In order to facilitate Web development, a number of languages blending database and programming language capabilities have recently been proposed [5, 12, 13, 17, 23]. Two well-known examples are LINQ [17], which extends .NET languages such as $\mathrm{C}$ \# or Visual Basic with querying primitives, and XQueryP [5], which extends the W3C XML Query language [2] with imperative features. Such languages aim at simplifying existing Web development practices, which typically rely on several different languages used at different tiers. The ability to blend data processing and programming capabilities frees the developer from the need to rely on low-level APIs, or use language embedding, for data access, but also raises significant challenges for compilers. Many database compilers already support expressive languages such as object-oriented languages [8], PL/SQL [11], XSLT [7], or XQuery [2], however, most of the work on query optimization has focused on languages without side effects. Side effects are essential in order to support key programming extensions, such as updates and variable assignment in XQueryP [5], or method calls in object languages [8].

In this paper, we propose techniques to adapt existing database compilers to support side effects in XQuery while

Permission to make digital or hard copies of all or part of this work for personal or classroom use is granted without fee provided that copies are not made or distributed for profit or commercial advantage and that copies bear this notice and the full citation on the first page. To copy otherwise, to republish, to post on servers or to redistribute to lists, requires prior specific permission and/or a fee.

Copyright 200X ACM X-XXXXX-XX-X/XX/XX $\ldots \$ 5.00$. preserving essential optimizations based on algebraic rewritings. Surprisingly, there has been very little work in that area in the past. One notable exception is [10] that uses a state monad [20] to support side effects in a nested-relational calculus. However, optimization at the algebraic, logical and physical level is not addressed. To the best of our knowledge, we provide the first treatment of side effects for a nestedrelational algebra. Due to space constraints, we limit our scope to updates applied during query evaluation, and leave procedural extensions (notably variable assignment) for future work. We start with a motivating example.

Usecase. Consider a simple scenario inspired by the sample retail application [1] provided with BEA's AquaLogic DSP [4]. This scenario assumes two XML data sources located at an on-line retailer site, named 'customers.xml' and 'orders.xml', made accessible by two XQuery functions, getCustomers and getUnconfirmedOrders. A customer can place orders, which are put on hold until they are confirmed by that customer. The application also maintains, through updates, access timestamps for customers data:

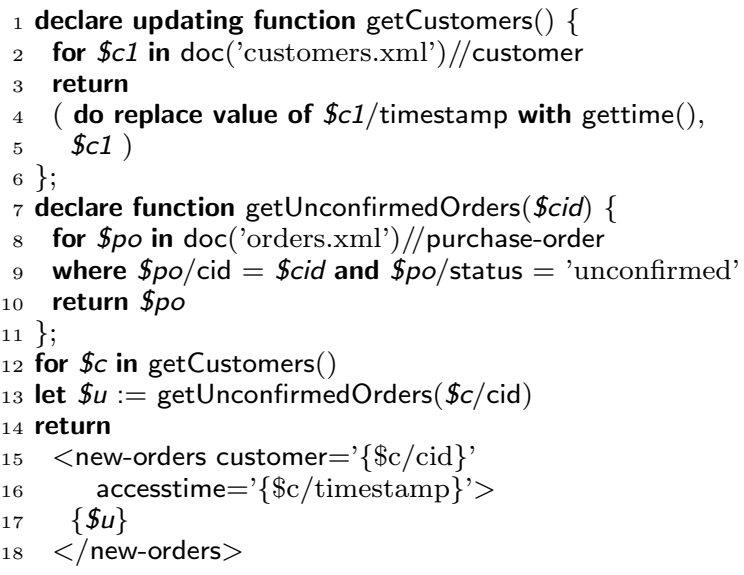

In the absence of the do replace update on line 4 , standard query optimizers would identify the query as a typical case of outer join between customers and orders (after function inlinining and using query unnesting techniques). In Section 3, we will see how similar nested queries can be optimized. We now give an overview of our approach.

Queries with side effects. We consider an extension of XQuery 1.0 [2] with simple update expressions. We adopt a semantics that imposes a strict left-to-right evaluation order and the immediate application of updates, in the style of 


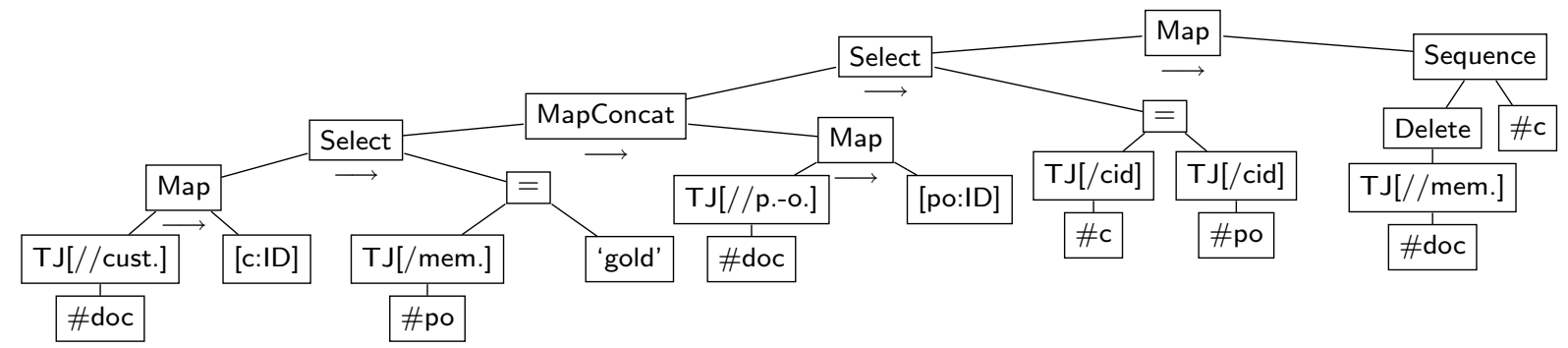

Figure 1: Original plan.

\section{XQueryP [5].}

We use a simple, artificial example, just to describe the technical challenges and illustrate our approach. The following query returns the set of orders for customers with gold membership, and if non-empty, removes all membership level information.

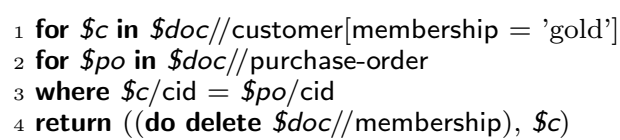

The language semantics specifies the following evaluation order: (i) retrieve the list of customer elements; (ii) expand the list of "gold" customers to a list of tuples with each customer paired with all possible purchase-orders; (iii) filter away the tuples that violate the predicate; (iv) for each tuple first remove all membership element nodes in the document and then return the customer element (the deletion has no effect from the second tuple onward). In this query, the optimizer should be able to use a join plan, since the updates occur only after all previous clauses are evaluated.

Logical optimization. The query is first compiled into a logical plan, shown in Figure 1, using a nested relational algebra with extensions for XML processing [19,21], as well as updates [13]. In addition to the standard nested-relational Select and Map, which applies its second operand to all items returned by its first operand, specific operators used in that example include TreeJoin (denoted TJ on the figure) for path navigation and Delete for node deletion. MapConcat corresponds to the traditional dependent product, and we use an arrow to indicate information flow from the first operand to the second. [a:ID] builds a one-field tuple, \#a selects the $a$ field from the input tuple. Again, the presence of updates requires the semantics of the algebra to specify an explicit evaluation order. We use an eager semantics in which every operator completely evaluates its first operand before starting the evaluation of the next operand.

In the absence of side effects, an optimizer would now turn the nested loop (MapConcat) operator into a Cartesian product, and would then combine it with the selection to get a join. Unfortunately, such a rewriting may be unsound in the presence of side effects. For instance, if the second Map operand of the MapConcat performs some insert, there must be as many inserts as there are items resulting from the first Map operand. We say that the operand is not idempotent: the store effects of repeated evaluations differ from those of a single evaluation, and hence, in this case, the nested loop must be retained. More generally, to be valid in presence of side effects, most classical rewritings require that some conditions are satisfied by the components of the rewritten plan. We show here that three side conditions suffice for this aim: commutativity, meaning that the evaluation order of two subplans can be exchanged, the aforementioned idempotency, and, in some cases, purity, indicating the absence of side effects. We also discuss how to infer the validity of these conditions for a plan, using static path analysis based on [14]. Using that technique, our optimizer can rewrite the plan above into the join-based plan shown in Figure 2.

Physical planning. At the physical level, one essential difference with a traditional compiler relates to pipelining. In a pure expression, the execution of the different operators can be freely interleaved (or pipelined), since their evaluation order is irrelevant. However, pipelining in the presence of updates requires additional care since the interleaved evaluation of steps from different operators may result in a later operator modifying data being accessed by an earlier operator. In the worst case, the semantics requires operators to materialize their input, making them behave as blocking operators. For example, a fully pipelined evaluation of the plan of Figure 2 would not be correct, since application of the Delete operator would interfere with the selection over the input customers.

Clearly, such blocking operators should be avoided when possible. We address this issue by controlling pipelining decisions during physical planning: each operator has both a version that materializes all its arguments (eager), and possibly additional versions that pipeline some of their arguments (lazy). The optimizer first produces a plan where all operators are eager, then uses rewrite rules to replace operators with lazy versions when it is safe to do so. In our example, the result is the plan in Figure 2, which only materializes on the input of the last Map operator, besides the materialization that is part of the hash-join algorithm.

We use three classes of side conditions for physical rewrite rules: purity, commutativity, and a notion of interleavability of two plans, which means that the effect of their interleaved evaluation is the same as that of a sequential evaluation. We show how such condition can be inferred using a variant of the path analysis used at the logical level for idempotency and commutativity inference.

Other approaches. Other semantics have been used for updates, such as the so-called snapshot semantics in the XQuery Update Facility [6], which delays update application until the end of the query. As was observed in [5], this approach is sufficient for typical database updates, but usually it is not expressive enough for programming purposes. Limitations to the locations where updates may occur have been suggested [5] to simplify compilation and optimization. However, such restrictions do not fully address the problem of optimization: as we will see at the end of Section 3, some 


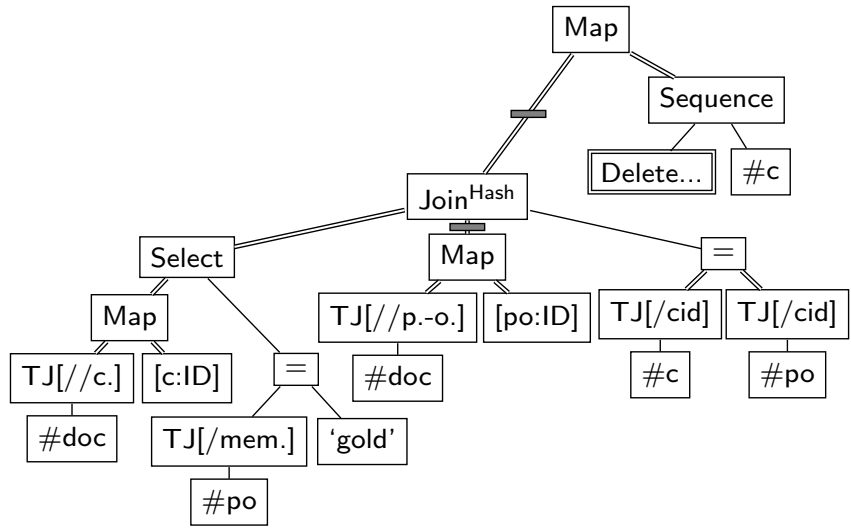

Figure 2: Optimized plan; hollow lines denote set arguments, a grey bar denotes materialization.

typical query unnesting optimizations require commutativity analysis even in the presence of some of those restrictions.

Contributions. The paper makes the following technical contributions.

- We extend a previously proposed algebra for XQuery plus side effects $[21,25]$ by providing the corresponding physical algebra.

- We study the side conditions needed to recover classical logical rewritings for join optimization and query unnesting in presence of side effects.

- We study pipelining in the context of a physical algebra with side effects, and provide similar side conditions that enable pipelining in query plans.

- We implemented the proposed algebra and side-effect analysis in an existing XQuery 1.0 compiler, and provide experimental result that validate the approach.

Plan. The paper is organized as follows. In Section 2 we introduce the algebra with side effects. In Section 3, we study logical rewritings in the presence of side effects. Section 4 presents changes necessary to the physical compiler. In Section 5, we present an experimental evaluation of the proposed approach. In Section 6, we review the related work. We conclude in Section 7.

\section{LANGUAGE AND ALGEBRA}

In this section we introduce the language and algebra used in the rest of the paper. We use XQuery extended with simple update primitives (delete, insert, replace), and the algebra of [21], extended with similar update operators. Both the semantics for the language and algebra follow a left-toright evaluation order with an eager evaluation of parameters. Also, both are fully compositional, i.e., updates may occur anywhere.

2.1 Definition (XQuery with updates). We use $E$ for expressions in the update language defined as follows:

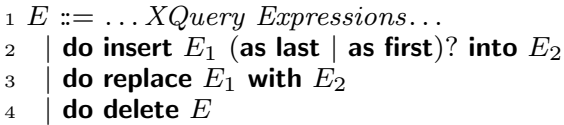

The three added update primitives respectively insert a new node in the document at a given location, replace an existing node by a new node, and delete a set of nodes.

The semantics of the language is the same as that of $\mathrm{XQueryP}$, i.e., expressions are evaluated in strict left-toright order, and updates are applied immediately. For instance, the following query declares a global variable $\$$ log, a function loglt which inserts its parameter into \$log and returns it, and a simple FLWOR expression calling loglt.

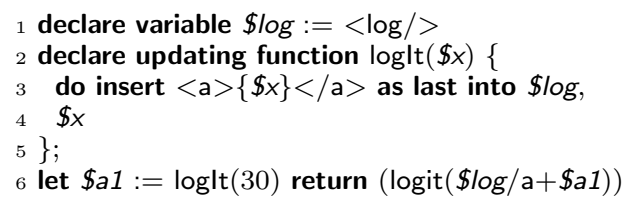

After evaluation, the $\$ \log$ variable contains the following XML value, in which two new a elements have been inserted in order: $<\log ><a>30</ a><a>60</ a></ \log >$.

Full semantics for such languages are typically described in terms of effects onto an XML store $[13,15,25]$. We use here the semantics of $[13,15]$, with one important exception in the case of FLWOR expressions, where we use a bulk semantics based on tuple streams: each clause produces a sequence of tuples which is fully evaluated before the next clause. As an example, consider the following query evaluated over the $\$ \log$ variable resulting from the previous query.

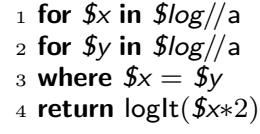

This query is an equi-join where the first two for clauses generate four $[x ; y]$ tuples, selecting two of them with the where clause, and finally adds 2 and 4 to the log. While the nested-for semantics of $[13,15]$ would alternate the evaluation of line 2 and line 4, the tuple-stream semantics is such that the logit function in line 4 would be only evaluated after the other clauses, hence does not interfere with the iteration in neither of the first two for clauses.

We now define an algebra to compile our language. It is based on the algebra of [21] extended with the operators Insert, Replace, and Delete. The algebra is defined on a logical data model whose values are either XML values, i.e., ordered sequences of items in the XQuery data model, or "tables", i.e., ordered sequences of tuples. A tuple is a record with fields containing XML values and written $\left[\mathrm{a}_{1}: \mathrm{v}_{1} ; \ldots ; \mathrm{a}_{n}: \mathrm{v}_{n}\right]$, where $\mathrm{a}_{1} \ldots \mathrm{a}_{n}$ are field names and $\mathrm{v}_{1} \ldots \mathrm{v}_{n}$ are the associated values. [] denotes the empty tuple. Sequences of tuples are used to compile the tuple stream of a FLWOR.

2.2 Definition (XML algebra with updates). Algebraic plans $p$ are terms constructed as follows:

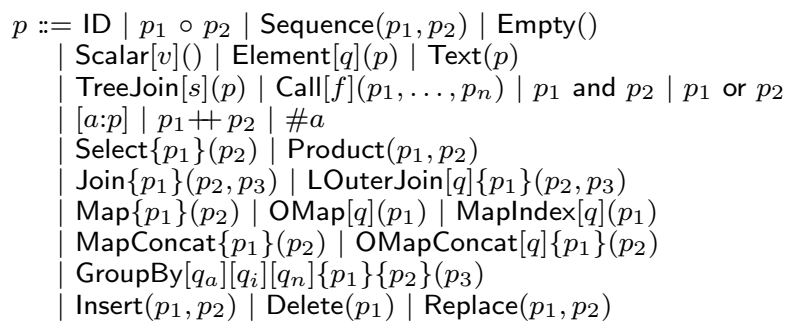

Each plan receives an input value, either an XML item or a tuple, when executed. For the operator subplans we use $\{p\}$ 
to indicate a dependent subplan, that gets its input from the subplans that follow in the parameter list $\left(p_{1}, \ldots, p_{n}\right)$ of the independent subplans, that process the input of the parent. $[q]$ is used for constant parameters.

ID returns the current input tuple, o passes the result of the right side plan as input to the left side (like function composition). Sequence, Empty, Scalar, Element, and Text, construct the corresponding XML values, TreeJoin extracts a sequence of nodes corresponding to an XML path step, Call invokes an XQuery function, and we have Boolean operators. Next follow basic operations on tuples, to construct singletons $[a: p]$, concatenate $p_{1}+p_{2}$, or access tuple fields $(\# a)$. Most of the other operators are based on a standard nested-relational algebra and should be familiar to the reader (Select, Product, Join, GroupBy, etc..). Map is the general functional map on sequences of tuples. MapConcat is a dependent product, equivalent to the DJoin operator of [18]. The OMap, OMapConcat, Maplndex and MaplndexStep are XQuery-specific map operators used during query unnesting [21]. Finally, we include the update operators. We allow infix notation for standard operators such as $=$.

The main difference with [21] is that operators are not always pure, but can change the state of the XML store being processed. This means that the algebra semantics must specify an evaluation order.

2.3 Definition (evaluation order for XML algebra with updates). The algebra operators have the semantics of [21], with the following evaluation order for their operands. Independent subplans are evaluated first, fully, once, and in the order in which they appear in the parameter list. The dependent parameter, if present, is evaluated next, once for each item in the result of the independent parameters, with the following exception: Composition $p_{1} \circ p_{2}$ evaluates the dependent parameter $p_{1}$ just once, on the whole result of $p_{2}$. Finally, the evaluation order of the two dependent parameters of GroupBy is specified in Section 3. For instance, by this definition, Join $\left\{p_{1}\right\}\left(p_{2}, p_{3}\right)$ first evaluates $p_{2}$, then $p_{3}$, takes their product, and finally it evaluates $p_{1}$ once for each tuple in the product.

2.4 Example. Here is the query plan obtained by compiling the query in the introduction.

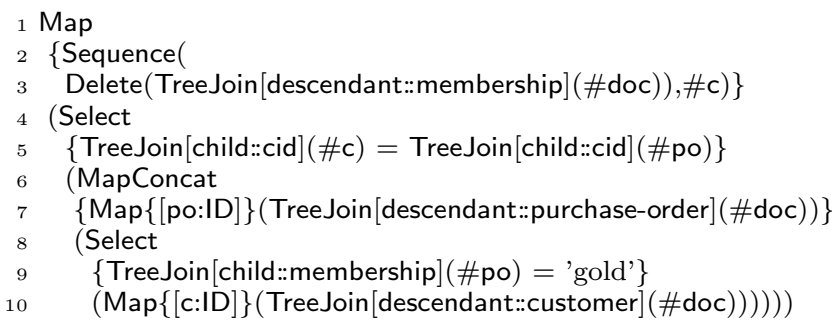

This plan corresponds to Figure 1, and uses tuples to encode variables bindings (tuples with field c and po are constructed through maps on lines 10 and 7), providing a direct representation of the tuple stream semantics of the language. The corresponding compilation rules are provided in $[18,21]$. Using the tuple stream makes this representation also amenable to join optimization and query unnesting. Join optimization in this example results in the following plan, which corresponds to Figure 2:

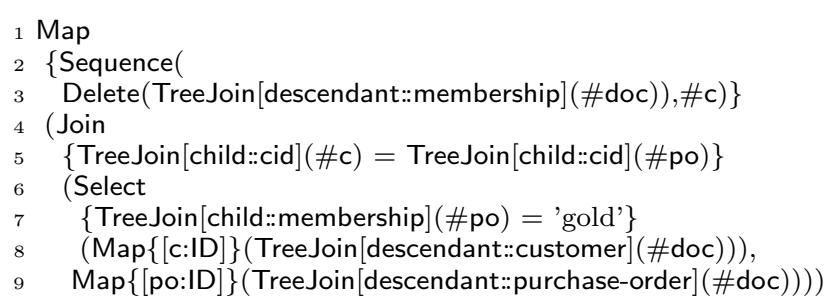

\section{LOGICAL OPTIMIZATIONS}

We present here our techniques to retain classical relational and nested-relational rewritings in the presence of side effects. Our version of such rewritings is reported in Figure 3. Unlike traditional relational equivalences, our rewritings are guarded by side conditions that depend on properties of the subplans, related to the presence of side effects. In the rest of the section, we discuss these properties and how the applicability conditions can be identified for each classical rewrite rule. While we discuss most of the rules in the text, for space reasons, we will have a full correctness discussion only for the (map through group-by) rule. We then show how these side conditions allow complex rewriting to happen even in presence of side effects in any position of the query plan.

Commutativity. Many rewrite rules change the evaluation order of some subplans. For example, the rules that push one operator inside the other, such as (push select) and (push product) in Figure 3, exchange the evaluation order of the plan that is pushed and the one that is traversed. The same happens with rule (merge select), for a subtler reason: assume we have three tuples, and $p_{2}$ is true for the first two; then, the left hand side evaluates the subplans in the order $p_{2}\left(t_{1}\right), p_{2}\left(t_{2}\right), p_{2}\left(t_{3}\right), p_{1}\left(t_{1}\right), p_{1}\left(t_{2}\right)$, while the right hand side alternates the two subplans, evaluating $p_{2}\left(t_{1}\right), p_{1}\left(t_{1}\right), p_{2}\left(t_{2}\right)$, $p_{1}\left(t_{2}\right), p_{2}\left(t_{3}\right)$. In both cases (push rules and merge select), the rewriting is sound if we can exchange any evaluation of $p_{1}(v)$ and $p_{2}\left(v^{\prime}\right)$, as expressed by commutativity.

3.1 Definition (commutativity). $p_{1}$ and $p_{2}$ commute, denoted $\operatorname{comm}\left(p_{1}, p_{2}\right)$, iff, for any input values $v$ and $v^{\prime}$, the resulting values and the store effects of $p_{1}(v)$ and $p_{2}\left(v^{\prime}\right)$ are the same independently of their evaluation order.

Observe that the (merge select) rule is sound since our and operator evaluates the parameters in order, and only evaluates the second when the first is true. If we used a strict and operator, which always evaluates both operands, the right hand side would evaluate $p_{1}$ on more tuples than the left hand side, hence we would need a further side condition requiring $p_{1}$ to be pure, i.e., free of side effects.

As observed in [14], commutativity is undecidable in general. To address that issue, we use the path analysis proposed in [14]: for every subplan $p$ we collect the set of $a c$ cessed paths $a(p)$ and the set of updated paths $u(p)$, which provide a static upper approximation of the nodes in the store that the plans reads and modifies. Two plans $p_{1}$ and $p_{2}$ commute if $u\left(p_{1}\right)$ is disjoint from $a\left(p_{2}\right) \cup u\left(p_{2}\right)$ and vice versa. Of course, this condition is always satisfied when the two plans are pure, since $u\left(p_{i}\right)$ is empty in that case. The main technical difference with [14] is that we extend the path language to deal with tuples. This extension is relatively straightforward and is not included here for space reasons. 


\begin{tabular}{|c|c|c|}
\hline MapConcat $\left\{p_{1}\right\}(\mathrm{ID}) \rightarrow p_{1}$ & & (remove map) \\
\hline MapConcat $\left\{p_{1}\right\}\left(p_{2}\right) \rightarrow \operatorname{Product}\left(p_{2}, p_{1}\right)$ & $\operatorname{indep}\left(p_{1}, p_{2}\right), \operatorname{idem}\left(p_{1}\right)$ & (insert product i) \\
\hline MapConcat $\left\{p_{1}\right\}\left(p_{2}\right) \rightarrow \operatorname{Product}\left(p_{2}, p_{1}\right)$ & $\operatorname{single}\left(p_{2}\right), \operatorname{indep}\left(p_{1}, p_{2}\right)$ & (insert product $s$ ) \\
\hline Select $\left\{p_{1}\right\}\left(\operatorname{Product}\left(p_{2}, p_{3}\right)\right) \rightarrow \operatorname{Join}\left\{p_{1}\right\}\left(p_{2}, p_{3}\right)$ & & (insert join) \\
\hline Select $\left\{p_{1}\right\}\left(\right.$ MapConcat $\left.\left\{p_{2}\right\}\left(p_{3}\right)\right) \rightarrow$ MapConcat $\left\{p_{2}\right\}\left(\operatorname{Select}\left\{p_{1}\right\}\left(p_{3}\right)\right)$ & $\operatorname{idem}\left(p_{1}\right), \operatorname{pure}\left(p_{2}\right), \operatorname{comm}\left(p_{1}, p_{2}\right), \operatorname{indep}\left(p_{1}, p_{2}\right)$ & ) (push select i) \\
\hline Select $\left\{p_{1}\right\}\left(\right.$ MapConcat $\left.\left\{p_{2}\right\}\left(p_{3}\right)\right) \rightarrow$ MapConcat $\left\{p_{2}\right\}\left(\operatorname{Select}\left\{p_{1}\right\}\left(p_{3}\right)\right)$ & $\operatorname{single}\left(p_{2}\right), \operatorname{pure}\left(p_{2}\right), \operatorname{comm}\left(p_{1}, p_{2}\right)$ & (push select s) \\
\hline Select $\left\{p_{1}\right\}\left(\right.$ Select $\left.\left\{p_{2}\right\}\left(p_{3}\right)\right) \rightarrow$ Select $\left\{p_{2}\right.$ and $\left.p_{1}\right\}\left(p_{3}\right)$ & $\operatorname{comm}\left(p_{1}, p_{2}\right)$ & (merge select) \\
\hline $\operatorname{MapConcat}\left\{p_{1}\right\}\left(\operatorname{Product}\left(p_{2}, p_{3}\right)\right) \rightarrow \operatorname{Product}\left(p_{2}, \operatorname{Map} \operatorname{Concat}\left\{p_{1}\right\}\left(p_{3}\right)\right)$ & $\operatorname{indep}\left(p_{1}, p_{2}\right), \operatorname{idem}\left(p_{1}\right), \operatorname{comm}\left(p_{1}, p_{2}\right)$ & (push product i) \\
\hline $\operatorname{MapConcat}\left\{p_{1}\right\}\left(\operatorname{Product}\left(p_{2}, p_{3}\right)\right) \rightarrow \operatorname{Product}\left(p_{2}, \operatorname{MapConcat}\left\{p_{1}\right\}\left(p_{3}\right)\right)$ & $\operatorname{indep}\left(p_{1}, p_{2}\right), \operatorname{single}\left(p_{2}\right), \operatorname{comm}\left(p_{1}, p_{2}\right)$ & (push product s) \\
\hline
\end{tabular}

Unnesting rewritings.

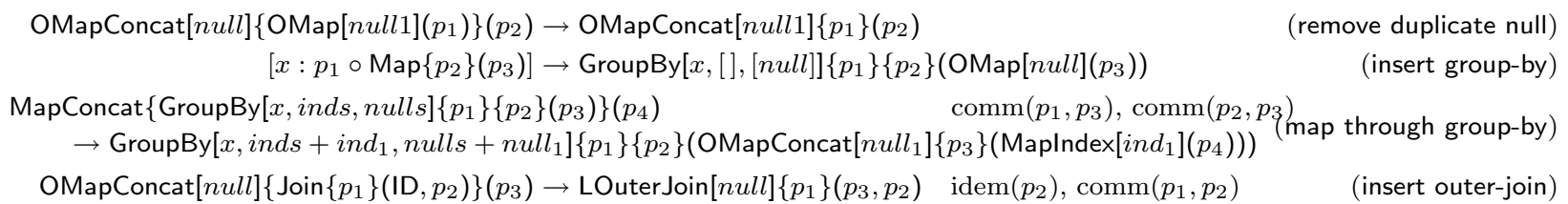

Figure 3: Logical algebraic rewritings.

Idempotency and Purity. While order exchange is important, the real efficiency boost comes from the ability to evaluate one expression once rather than many times, or to skip evaluation altogether. The simplest, and arguably most important, example is the (insert product i) rewriting in Figure 3 . The rewritten version evaluates $p_{1}$ once, while the original version evaluates it as many times as there are input tuples in $p_{2}$. This is a key rewriting, as it also clears the way for the introduction of joins in the plan. As discussed in the introduction, this is safe only if $p_{1}$ is idempotent, according to the definition below.

Idempotency is also needed in the push... i rules, since they also reduce the number of times $p_{1}$ is evaluated, by a factor that is equal to the size of $p_{2}$. When $p_{2}$ is a singleton, however, there is no difference in the number of times $p_{1}$ is evaluated, and there is no need to check idempotency (rules (push...s) and (insert product s)).

3.2 Definition (idempotency). $p$ is said to be idempotent, denoted $\operatorname{idem}(p)$, iff, for any input value $v$, two evaluations of $p(v)$ result in the same effects and value as one evaluation of $p(v)$.

While idempotency is undecidable in general, we approximate it using path analysis: a plan $p$ is idempotent if $a(p)$ is disjoint from $u(p)$, so that its second evaluation will produce the same results as the first, and $p$ performs no insert operation. Again, every pure plan satisfies this test.

It is important to note that checking for idempotency only works if the cardinality of evaluation is reduced but the corresponding plan is evaluated at least once. Considering the (push select) rules, we note that $p_{2}$ is not applied, in the right side, to the tuples that do not satisfy $p_{1}$, while it is applied to every tuple in the left side. For that reason, we need to check for purity of $p_{2}$; purity inference is trivial.

Standard properties. In addition to properties related to side effects, we also use a couple of more standard properties, namely independence (whether a plan depends on the tuple fields returned or added by a particular plan), and typing (notably whether a plan returns a singleton tuple) properties. They are defined as follows.
3.3 Definition (independence). $p_{1}$ is independent of $p_{2}$, denoted indep $\left(p_{1}, p_{2}\right)$, iff $p_{1}$ does not access the fields created by $p_{2}$.

3.4 Definition (singleton). $p$ is said to be a singleton plan, denoted single $(p)$, iff $p$ always returns a single tuple.

Note that both independence and singleton properties can be checked through static typing at the algebraic plan level.

\section{Query unnesting.}

3.5 Scenario (Implicit grouping). In the rest of the section, we focus on how the above framework applies to a typical query unnesting scenario, and we discuss the (map through group-by) rule. Let us consider the following simple class of nested queries.

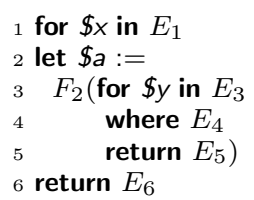

where, $E_{1}, E_{3}, E_{5}, E_{6}$ are arbitrary XQueryP expressions, $F_{2}$ is an aggregate function, and $E_{4}$ is a join predicate between $\$ x$ and $\$ y$. This nesting form corresponds to the implicit grouping pattern in the sense of [18].

After function inlining and some simple syntactic rewrites, the first example in the introduction is an instance of this nesting form. For exposition purposes, we use a version of that query with additional side effects, in which the system maintains logs containing the number of unconfirmed orders per customer (using logcount) and a backup copy of those individual orders (using logord):

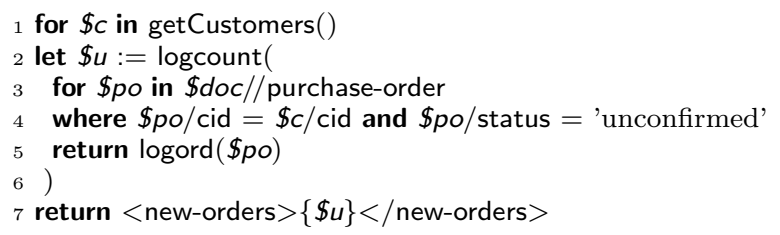


In this example, $E_{1}$ and $E_{3}$ correspond to accessing customers and orders, respectively. $F_{2}$ is the logcount function, $E_{4}$ is the conjunctive join predicate, and $E_{5}, E_{6}$ are, respectively, the inner and outer return clauses. Our goal is to be able to unnest the query using traditional rewritings $[9,18,19]$. Indeed, for this query, we can reuse the same sequence of rewriting rules as were used in [21]. The query is first compiled into the following plan.

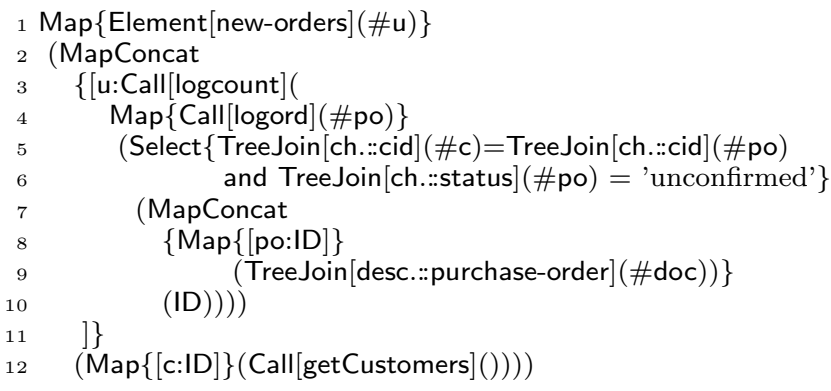

The [u:Call[logcount](..)] subplan (lines 4-10) corresponds to the let $\$ u$ clause and nested FLWOR in the query, and the MapConcat operator evaluates it once for each customer. The plan is unnested into the plan below. The unnested plan, instead of using a nested loop, performs an outer join followed by a single GroupBy that evaluates logord for each unconfirmed order, and logcount for each group of unconfirmed orders (the detailed semantics of GroupBy is explained below).

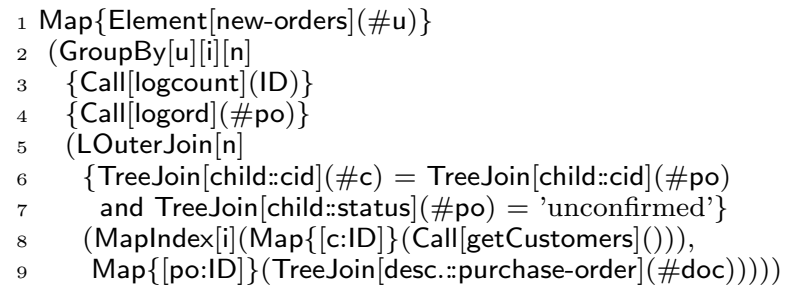

The unnesting technique is standard (see [21], for example, for details), apart from the presence of side effects and rule conditions. Apart from bookkeeping rewritings, the main sequence is: (insert group-by) introduces an empty GroupBy instead of tuple construction on line 3; (map through groupby) pushes the MapConcat through the GroupBy resulting in the proper grouping over $\mathrm{i}$, which is an index used to keep track of sequence order; the inner MapConcat operator on line 7 is turned into a trivial Cartesian product, which combined with the Select yields a Join; finally, after simplifying the combination of OMapConcat and OMap into an OMapConcat, (insert outer-join) is used to introduce the final LOuterJoin.

Most of the side conditions for those rewritings are based on the same principles that we explained earlier in the section. The (map through group-by) rewrite, though, is more complex, and worth explaining (we discuss here the side conditions only, since the rule is not new [21]). We first define the semantics of GroupBy $[a]\left[k_{1} \ldots k_{m}\right][n]\left\{p_{1}\right\}\left\{p_{2}\right\}\left(p_{3}\right)$, and, specifically, the order in which the dependent subplans $p_{2}$ and $p_{1}$ are evaluated. The parameter $k_{1} \ldots k_{m}$ is the set of grouping fields, $a$ is the field name for the new result of the aggregation function, and $n$ is the null field, that we ignore here, since it adds nothing to the discussion of side effects and conditions. The semantics is specified as follows.

1. First $p_{3}$ is evaluated, resulting in a table $T$, and the sequence of grouping keys is computed; this set is the projection of $T$ on $k_{1} \ldots k_{m}$.

2. Then for each key tuple $k t$ : (a) $p_{2}$ is computed for each tuple $t_{1}^{k t} \ldots t_{n}^{k t}$ of $T$ that coincides with $k t$ over $k_{1} \ldots k_{m}$, (b) the aggregate value $a v$ is computed by applying $p_{1}$ to the set $\left\{p_{2}\left(t_{1}^{k t}\right), \ldots, p_{2}\left(t_{n}^{k t}\right)\right\}$, and the tuple $[a: a v]+k t$ is emitted; hence, the evaluations of $p_{2}$ and $p_{1}$ are alternated.

The right side of the (map through group-by) rule enriches each tuple of $p_{4}$ with an index field $i n d_{1}$, computes the (outer) dependent product of $p_{3}$ and $p_{4}+i n d_{1}$, and then applies a GroupBy; the difference between OMapConcat and MapConcat is not relevant to our discussion here.

We first observe that on both sides of the rewriting rule, $p_{4}$ is evaluated before any other plan so we don't need any condition involving $p_{4}$.

A second simple observation is that after rewriting, all evaluations of $p_{3}$ occur before those of $p_{1}$ and $p_{2}$, while its evaluations were originally alternating with those of $p_{1}$ and $p_{2}$. Hence $p_{3}$ must commute with both $p_{1}$ and $p_{2}$.

The remaining question is whether $p_{1}$ and $p_{2}$ need to commute or be idempotent. The answer follows from the specific semantic details of GroupBy: the sequence of $p_{1}$ and $p_{2}$ evaluations is exactly the same in the two cases. Let us illustrate this point on our example. The following shows the plan just before the (map through group-by) rewrite is applied.

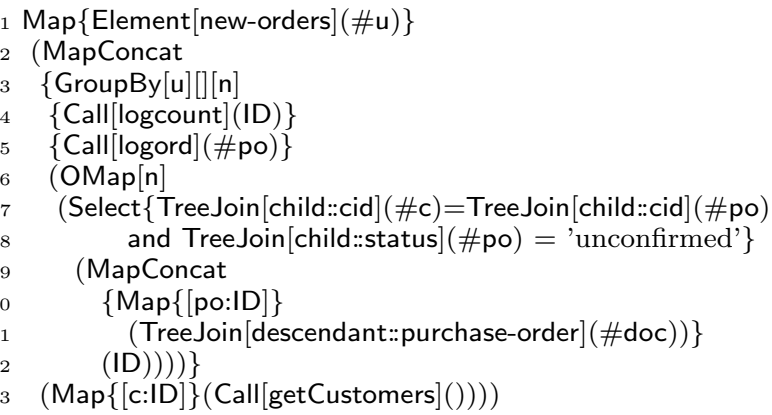

Both $p_{1}$ (logcount) and $p_{2}$ (logord) have side effects. We use $a_{1}, \ldots, a_{n}$ to denote updates performed by the logcount function, and $b_{1}, \ldots, b_{n}$ to denote updates performed by the logord function.

When this plan is processed, first $p_{4}$ is fully evaluated, and then, for each of the bindings of c, the GroupBy is evaluated and the updates applied. Let's assume the group-by results in three groups, the first and last with two matching tuples, and the second one with one matching tuple. The following table contains one subtable for each tuple generated by $p_{4}$. Each subtable contains: (i) the table (with fields c, po, and n) produced by $p_{3}$, (ii) The side effects produced by $p_{2}$ applied to each element in the output of $p_{3}$ (indicated $a s b_{i}$ ), and then by $p_{1}$ applied to the whole output of $p_{2}$ (indicated as $\mathrm{a}_{i}$ ), (iii) the final value produced by $p_{1}$.

\begin{tabular}{|ccc||c||c|}
\hline $\mathrm{c}$ & po & $\mathrm{n}$ & effects & $u$ \\
\hline \hline$c_{1}$ & $p o_{1}$ & $f$ & \multirow{2}{*}{$\mathrm{b}_{1}, \mathrm{~b}_{2}, \mathrm{a}_{1}$} & 2 \\
$c_{1}$ & $p o_{2}$ & $f$ & \\
\hline \hline$c_{2}$ & $p o_{3}$ & $f$ & $\mathrm{~b}_{3}, \mathrm{a}_{2}$ & 1 \\
\hline \hline$c_{3}$ & $p o_{4}$ & $f$ & $\mathrm{~b}_{4}, \mathrm{~b}_{5}, \mathrm{a}_{3}$ & 2 \\
$c_{3}$ & $p o_{5}$ & $f$ & \\
\hline
\end{tabular}


Thus the final sequence of effects is $\left(b_{1}, b_{2}, a_{1}, b_{3}, a_{2}, b_{4}, b_{5}\right.$, $\left.a_{3}\right)$. Applying the rewriting, which pushes the MapConcat through the GroupBy, leads to the following plan:

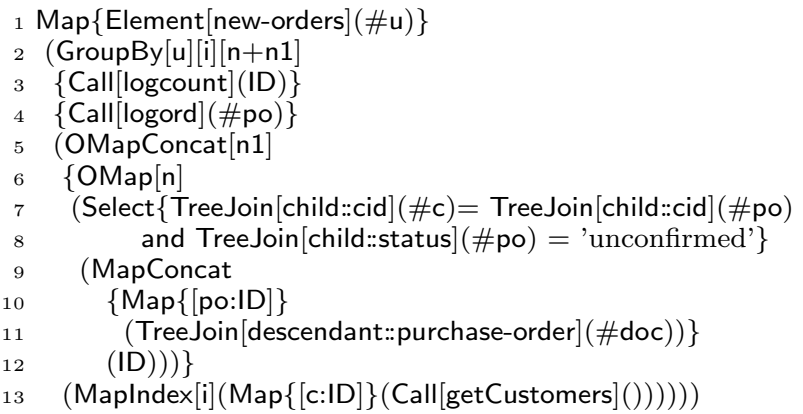

inside which the OMapConcat will be merged with the OMap and then rewritten into a LOuterJoin. Note that the effects for $p_{3}$ are all applied before the GroupBy is evaluated. (The GroupBy operator groups on the i field, and, for each group, applies $p_{2}$, followed by $p_{3}$.) This results in the following table and effects.

\begin{tabular}{|ccc||c|}
\hline $\mathrm{c}$ & $\mathrm{i}$ & $\mathrm{u}$ & effects \\
\hline \hline$c_{1}$ & 1 & 2 & $\mathrm{~b}_{1}, \mathrm{~b}_{2}, \mathrm{a}_{1}$ \\
$c_{2}$ & 2 & 1 & $\mathrm{~b}_{3}, \mathrm{a}_{2}$ \\
$c_{3}$ & 3 & 2 & $\mathrm{~b}_{4}, \mathrm{~b}_{5}, \mathrm{a}_{3}$ \\
\hline
\end{tabular}

The final sequence of effects is $\left(b_{1}, b_{2}, a_{1}, b_{3}, a_{2}, b_{4}, b_{5}, a_{3}\right)$. The relative order of effects between $p_{1}$ and $p_{2}$ is unchanged.

Hence, in order to apply (map through group-by) to our query, we have only to verify that $p_{1}$ and $p_{2}$ commute with $p_{3}$. With reference to the general query, we can easily verify that $p_{1}$ corresponds to $F_{2}, p_{2}$ to $E_{5}$, and $p_{3}$ to $E_{3}$ restricted by $E_{4}$, hence this rewrite rule is applied when $F_{2}$ and $E_{5}$ do not interfere with $E_{3}$ and $E_{4}$. No other rule applied during the unnesting process has side effect related conditions, apart from the crucial one, (insert outer-join). Through a similar analysis, we can verify that it requires idempotency of $E_{3}$ and commutativity of $E_{3}$ and $E_{4}$.

Discussion. Applying that same sequence of rewritings to the nested form in Scenario 3.5, we can see that the complete set of conditions which must be checked is as follows.

- idempotency: idem $\left(E_{3}\right)$

- commutativity: $\operatorname{comm}\left(F_{2}, E_{4}\right), \operatorname{comm}\left(F_{2}, E_{3}\right)$, $\operatorname{comm}\left(E_{5}, E_{4}\right), \operatorname{comm}\left(E_{5}, E_{3}\right), \operatorname{comm}\left(E_{3}, E_{4}\right)$.

Based on those conditions, we can make the following general remarks.

- If commutativity-based side conditions were substituted with purity requests, the presence of a side effect in any position apart from $E_{1}$ and $E_{6}$ would prevent unnesting; on the other side, if one is ready to do commutativity (and idempotency) analysis, then optimization would be compatible with the presence of side effects in any position, provided that they do not interfere with the rest of the query; hence, commutativity is buying us a lot.

- We expect that updating and accessing the same data in the internal parts of a query will be quite rare in practice, so that the commutativity side conditions should be satisfied in the vast majority of situations.
- Our rules only impose idempotency for $E_{3}$, and this seems minimal, because some of the essence of join optimization is to evaluate $E_{3}$ only once.

Finally, we come back to the issue of language restrictions. Remember that the XQueryP [5] proposal does not allow updates in any other clause but the return of a FLWOR expression. Still, the typical nesting pattern we studied in this section can be written solely with side effects in return clauses. Here is the example we used, written in that form.

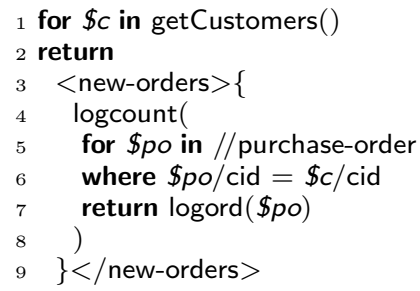

As before, producing the proper unnested plan in this example requires checking commutativity between the log functions and the subquery that accesses the purchase order. Hence, the XQueryP restriction, although is quite strong, is still not sufficient to avoid the need for techniques like those we present here.

\section{PHYSICAL PLANNING}

In this section, we look at the impact of the presence of side effects on code selection (picking up physical algorithms), and on pipelining. As we have seen in the introduction, pipelining can interact heavily with the presence of side effects. Other aspects related to cost-based optimization are beyond the scope of our work at this point. We first give an overview of the proposed approach.

Overview. We represent physical operators as logical operators annotated with two parameters, as in Join ${ }_{\mathrm{SEE}}^{\mathrm{NL}}$ (NL means nested loop) or Join SLE $_{\text {Sash }}$; the superscript indicates the algorithm being used, while the subscript indicates which arguments are evaluated eagerly $(\mathrm{E})$, which are evaluated lazily (L), and which return a singleton (S), like in the case of the Join predicate. The algorithm parameter is missing when we only have one implementation, as in SelectSE.

To produce a physical plan, our compiler first generates a plan that only uses default algorithms and eager operators, such as Join $_{\mathrm{SEE}}^{\mathrm{NL}}$, since this combination is guaranteed to respect the semantics in presence of side effects. Then, a set of rewrite rules is applied which allows better algorithms to be selected. Finally, another set of rewrite rules is applied which allows eager arguments to be substituted with lazy arguments, hence reducing the amount of data to be materialized. As for logical rewritings, rules in both sets are based on side conditions that are related to the presence of side effects. Note that these rules only change aspects of the evaluation for each operator and never modify the shape of the query plan, The full set of rewritings is given in Figure 4. Before we explain the side conditions in details, let us first illustrate how they are used.

Consider the optimized plan in Example 2.4 (Figure 2). The compiler first produces the following plan. In that plan, every operator has a default implementation and every input is eagerly evaluated (i.e., materialized), as indicated by the corresponding annotations. 


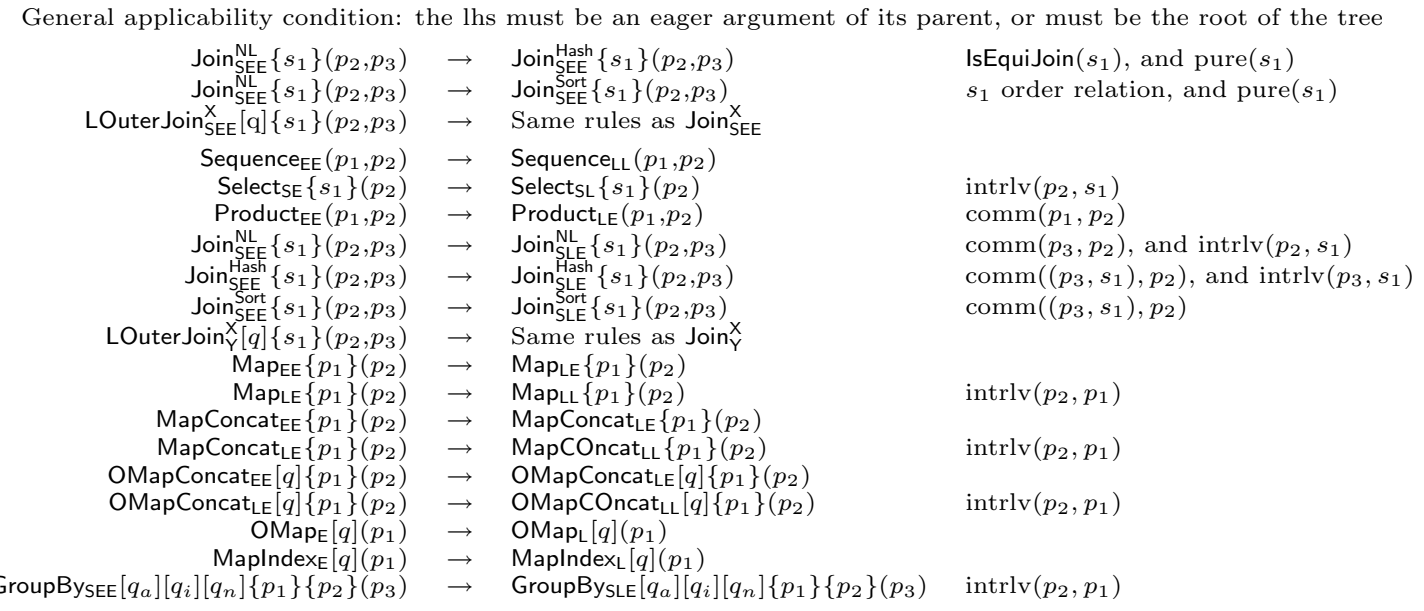

Figure 4: Physical algebraic rewritings.

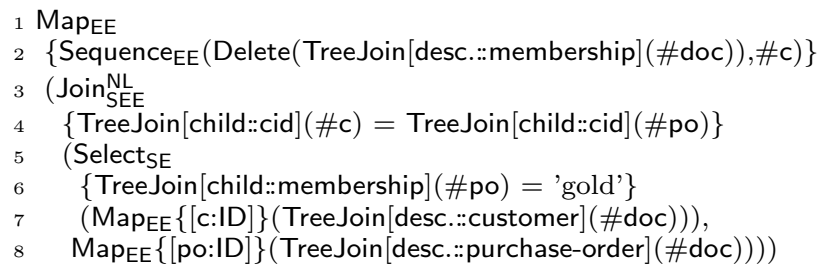

Join $\mathrm{NEE}_{\mathrm{SE}}$ can be rewritten as a hash join, since its first argument is pure and contains an equality, as follows.

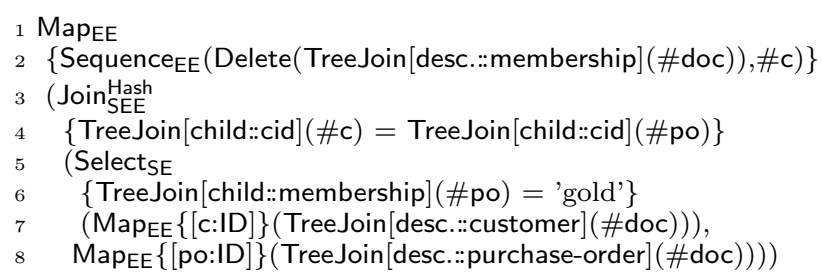

Now, one specific challenge in the context of such physical plans is that rewritings in various parts of the plan may not be independent. For instance, let us assume we first apply a rewrite rule that introduces laziness on the final Map. This rewriting is sound since the branch of the join that accesses customers' membership status is evaluated eagerly, and the final delete operator does not modify any of the data used by the join predicate. The results is the following plan.

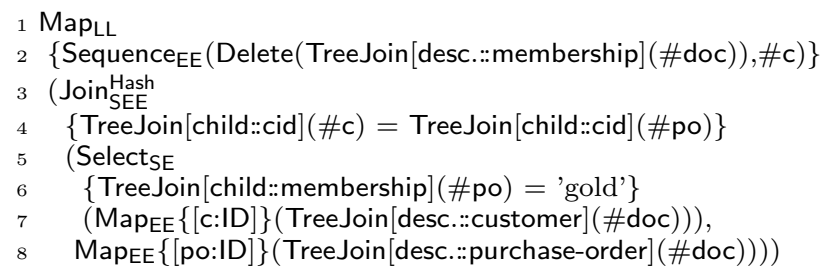

After this rewrite, however, we can no longer pipeline the left branch of the join that accesses customers, since the evaluation of the Join input and the outer Map would be interleaved, resulting in membership information being deleted while still processing customer information. In the absence of a cost model, we solve this problem applying the rules for pipelining introduction in a bottom-up fashion. I.e.,, rules are always applied in a context of an eager argument or at the root of the tree. This strategy is simple and seems to be a reasonable starting point since it follows the aim of pipelining "as long as possible". With this bottom-up application approach, the two inner Map operators and Select operators are rewritten as lazy since their input plans are pure. The Join ${ }_{\mathrm{SEE}}^{\text {Hash }}$ is rewritten to pipeline its left branch. The Sequence EE has no side conditions, hence it is applied. Moving to the root, the outer Map $\mathrm{P}_{\mathrm{EE}}$ to MapLE comes for free, and we get the following result where only the right branch of the join, and the input of the outer map are materialized as in Figure 2.

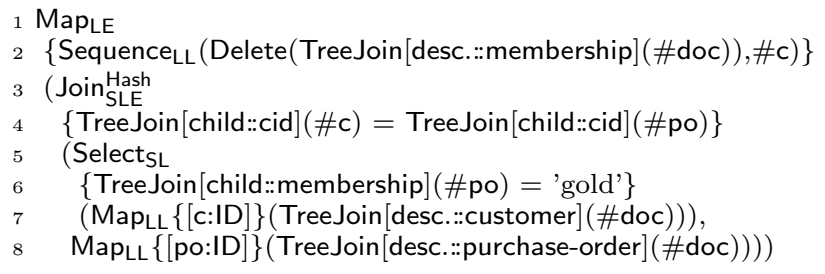

Rewrite rules. Not all the operators are subject to eager/lazy optimization. Specifically, we do not need to consider the operators whose output is a singleton, (e.g., Empty, Scalar, Element, TreeJoin). Figure 4 shows the necessary rules for the remaining operators.

To discuss those rules, we need a notation to define the semantics of physical operators. To this aim, we use the yield syntax made popular by $\mathrm{C} \# 2.0$. In our notation, $\mathrm{P} \mathrm{x}_{1} \ldots \mathrm{x}_{n}$ $:=$ new iterator $\{$ block $\}$ builds an object $\mathrm{P}$ with two methods, MoveNext and Current. P.Current returns the value of an object field named Current, which is initially null, and which is updated by P.MoveNext. P.MoveNext evaluates block until it finds a "yield value" statement, then stores value into P.Current, saves the execution state of the block, and returns true. The next time P.MoveNext is called, it starts from the execution state where it stopped the previous time; when it finds no more yield value to execute, it returns false, and sets P.Current to null. For example, consider the following iterator:

${ }_{1}$ PList $:=$ new iterator $\{$ foreach $(x$ in $(1,2))\{$ yield $x\}$; yield 3$\}$

PList.MoveNext returns true three times, setting Current to 1,2 , and 3 , and returns false when called for the fourth time onwards. PList.Current evaluates to 1,2 and 3 after the first three calls to PList.MoveNext, and returns null before the first call and after the fourth. Using such syntax, we can 
easily write the semantics for the eager and lazy versions of our operators.

We start with the Cartesian product Productxx operators. The foreach ( $\mathrm{t}$ in Expr) \{block\} statement builds a new iterator $\mathbf{I}=$ Expr, and evaluates block once for each successful invocation of I.MoveNext, binding t to I.Current. Every plan has a parameter $\mathrm{x}$, used to transmit context information, for example to a subplan $p_{1}$ that depends on the output of another subplan $\mathrm{p}_{2}$, as in Join $\times x\left\{\mathrm{p}_{1}\right\}\left(\mathrm{p}_{2}, \mathrm{p}_{3}\right)$. Most operators just pass $x$ to their subplans, while the field lookup (\#a) and navigation (TreeJoin $[$ path]) operators actually use it. (In a typical implementation, the parameter is passed to a plan $\mathrm{P}$ using a P.Open $(\mathrm{x})$ operation, but this is irrelevant here.)

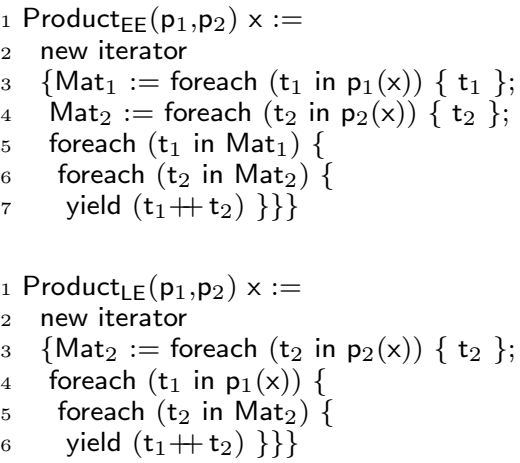

Both operators support the MoveNext-Current interface, but Product $_{\mathrm{EE}}$ implements the eager semantics by materializing

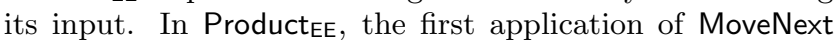
(hereafter, MN) builds both $\mathrm{Mat}_{1}$ and $\mathrm{Mat}_{2}$, and returns the control to the caller when the first output tuple is built (using tuple concatenation $\mathrm{t}_{1}+\mathrm{t}_{2}$ ); the next call will start from that point. In Product ${ }_{\mathrm{LE}}$, the first application of $\mathrm{MN}$ builds $\mathrm{Mat}_{2}$ and calls $\mathrm{p}_{1}(\mathrm{x})$.MN just once; the next call to $\mathrm{p}_{1}(\mathrm{x}) \cdot \mathrm{MN}$, implicit in the foreach $\left(\mathrm{t}_{1}\right.$ in $\left.\mathrm{p}_{1}(\mathrm{x})\right)$ notation, will be caused by the next call to $\left(\operatorname{Product}_{\mathrm{EE}}\left(\mathrm{p}_{1}, \mathrm{p}_{2}\right) \mathrm{x}\right) \cdot \mathrm{MN}$. In other words, Product $\mathrm{LE}_{\mathrm{L}}$ pipelines on its left input, as in a traditional side effect free relational implementation.

We need to define one more notion before talking about correctness of the rewritings. We define a consecutive evaluation of a plan $\mathrm{P}$ to be a sequence of calls to P.MoveNext, where the last call returns false and no other store action is interleaved between two calls (consecutive). We define the total result of such an evaluation of a plan $\mathrm{P}$ as the pair of the ordered sequence of all the values (the value result) and the store effect (the store result) that it produces.

Looking at the rule for product on Figure 4, the total result of $\operatorname{Product}_{\mathrm{EE}}\left(\mathrm{p}_{1}, \mathrm{p}_{2}\right) \times$ is obtained through a sequence of calls $\mathrm{p}_{1}(\mathrm{x}) \cdot \mathrm{MN}, \ldots, \mathrm{p}_{1}(\mathrm{x}) \cdot \mathrm{MN}$, followed by $\mathrm{p}_{2}(\mathrm{x}) \cdot \mathrm{MN}, \ldots$, $\mathrm{p}_{2}(\mathrm{x}) . \mathrm{MN}$, while the Product $\mathrm{LE}_{\mathrm{L}}$ version uses the inverse sequence $\mathrm{p}_{2}(\mathrm{x}) \cdot \mathrm{MN}, \ldots, \mathrm{p}_{2}(\mathrm{x}) \cdot \mathrm{MN}, \mathrm{p}_{1}(\mathrm{x}) \cdot \mathrm{MN}, \ldots, \mathrm{p}_{1}(\mathrm{x}) \cdot \mathrm{MN}$.

From this observation we deduce the soundness of our first rewrite rule, where commutativity $\operatorname{comm}\left(\mathrm{p}_{1}, \mathrm{p}_{2}\right)$ is formally defined below.

If (a) $\operatorname{Product}_{\mathrm{EE}}\left(p_{1}, p_{2}\right)$ is an eagerly evaluated subplan in the context $P[]$ and (b) $\operatorname{comm}\left(p_{1}, p_{2}\right)$, then $P\left[\operatorname{Product}_{\mathrm{EE}}\left(p_{1}, p_{2}\right)\right] \rightarrow P\left[\operatorname{Product}_{\mathrm{LE}}\left(p_{1}, p_{2}\right)\right]$

To understand (a), consider that commutativity of $p_{1}$ and $p_{2}$ ensures that the total results of $\operatorname{Product} \operatorname{te}\left(p_{1}, p_{2}\right)$ and Product $_{\mathrm{LE}}\left(p_{1}, p_{2}\right)$ coincide, but still the partial effects of invoking, for example, the first MoveNext is quite different in the two cases: the EE operator will fully evaluate its subplans, while the LE operator will just invoke the second one. When Product $\mathrm{EE}\left(p_{1}, p_{2}\right)$ is an $\mathrm{E}$ operand for its parent, we only care about the total result, hence the rewriting is sound.

Let us now look at the join operator. Here is a typical description of the nested-loop join using our iterator syntax.

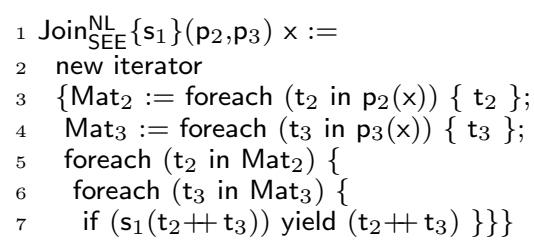

As usual, hash join requires $s_{1}$ to contain an equality condition. As was observed in [21], the situation is slightly more complex with XQuery because of the existential semantics of equality and because equality depends on types. This has little to do with side effects, hence we adopt here a basic approach: our hash join algorithm assumes that $\mathrm{s}_{1}$ has the "equijoin shape" $s_{11} \wedge\left(s_{12}=s_{13}\right)$, where $s_{12}$ and $s_{13}$ only depend on tuple fields produced, respectively, by $\mathrm{p}_{2}$ and $\mathrm{p}_{3}$ (as in the plan in Figure 2). We use a predicate IsEquiJoin $\left(s_{1}\right)$ to check this condition and a function DecomposeEquiJoin $\left(\mathrm{s}_{1}\right)$ to decompose $\mathrm{s}_{1}$ (in Figure 4). Here is the corresponding implementation. Note that the creation of the hashtable (lines 5 to 6 ) amounts to materialization.

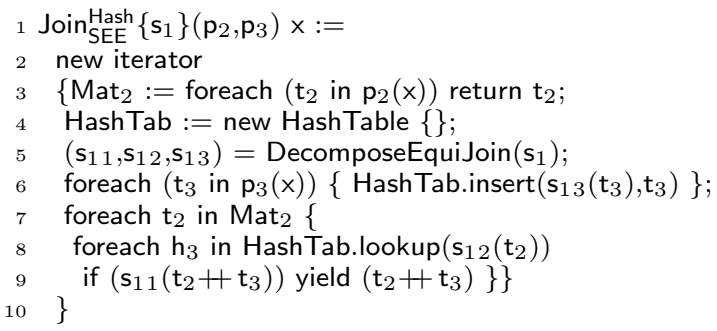

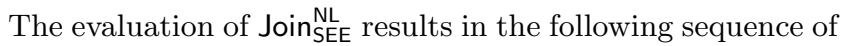
MoveNext calls: $\mathrm{p}_{2}, \ldots, \mathrm{p}_{2}, \mathrm{p}_{3}, \ldots, \mathrm{p}_{3}, \mathrm{~s}_{1}, \ldots, \mathrm{s}_{1}$. The call sequence for Join HEE $_{\mathrm{HEE}}$ may be represented as follows: $\mathrm{p}_{2}, \ldots$, $\mathrm{p}_{2}, \mathrm{p}_{3}, \ldots, \mathrm{p}_{3}, \mathrm{~s}_{13}, \ldots, \mathrm{s}_{13}, \mathrm{~s}_{12}, \mathrm{~s}_{11}, \ldots, \mathrm{s}_{11}, \mathrm{~s}_{12}, \mathrm{~s}_{11}, \ldots$, $\mathbf{s}_{11}$. The only difference is that $\mathbf{s}_{1}$ evaluation is decomposed, and $s_{1}$ is not applied to every tuple pair. We hence require $\mathrm{s}_{1}$ to be pure, and have the first rule in Figure 4.

Finally, the lazy version of the hash join (Join SLE $_{\text {Hash }}$ ) only differs in that it omits the materialization of the left branch (line 3 ). In this case, the call sequence is $\mathrm{p}_{3}, \mathrm{~s}_{13}, \ldots, \mathrm{p}_{3}$, $\mathrm{s}_{13},\left(\mathrm{p}_{2}, \mathrm{~s}_{12}, \mathrm{~s}_{11}, \ldots, \mathrm{s}_{11}, \ldots, \mathrm{p}_{2}, \mathrm{~s}_{12}, \mathrm{~s}_{11}, \ldots, \mathrm{s}_{11}\right)$. To rewrite Join ${ }_{S E E}^{\text {Hash into Join }}{ }_{S L E}^{\text {Hash }}$ we first assume that $p_{2}$ and $p_{3}$ commute, hence the trace of the first is equivalent to $\mathrm{p}_{3}, \ldots$, $\mathrm{p}_{3}, \mathrm{p}_{2}, \ldots, \mathrm{p}_{2}, \mathrm{~s}_{13}, \ldots, \mathrm{s}_{13}, \mathrm{~s}_{12}, \mathrm{~s}_{11}, \ldots, \mathrm{s}_{11}, \mathrm{~s}_{12}, \mathrm{~s}_{11}, \ldots$, $\mathrm{s}_{11}$. Equivalence follows if we further assume that $\mathrm{s}_{12}$ and $\mathrm{s}_{11}$ calls can be commuted with $\mathrm{p}_{2}$, and that $\mathrm{s}_{13}$ calls can be commuted with both $\mathrm{p}_{3}$ and $\mathrm{p}_{2}$. This sounds complex, but amounts to inferring that all of $\mathrm{p}_{2}, \mathrm{p}_{3}$ and $\mathrm{s}_{1}$ are mutually read-write disjoint.

Interleavability. Before presenting the interleavability side condition, it is better to introduce a couple of definitions that will allow us to be more precise about commutativity as well. We first define an interleaved evaluation of $p_{1}$ and $p_{2}$ as a sequence of calls to $p_{1}$.MN interleaved with calls to $p_{2} . \mathrm{MN}$ (and nothing else), where the last calls $p_{1} . \mathrm{MN}$ and $p_{2}$.MN both return false. The consecutive evaluation of one 
plan followed by the consecutive evaluation of the other is hence a special case of interleaved evaluation.

4.1 Definition (Commutativity). $\operatorname{comm}\left(p_{1}, p_{2}\right)$ iff, for any interleaved evaluation of $p_{1}$ and $p_{2}$ that start from a given store: (a) the store result is the same; (b) the value result of $p_{1}$ is the same; (c) the value result of $p_{2}$ is the same.

This notion of commutativity is quite stronger than the logical one, since we may actually interleave steps of one operator with steps of the other. Most of our rewrite rules would be consistent if we adopted a weaker notion of commutativity, where we only require that the consecutive evaluation of $p_{2}$ after $p_{1}$ has the same result as the consecutive evaluation of $p_{1}$ after $p_{2}$. However, we statically infer $\operatorname{comm}\left(p_{1}, p_{2}\right)$ from read-write disjointness of $p_{1}$ and $p_{2}$, and both versions of commutativity follow from such disjointness, hence we just consider the strong definition above. Besides purity and commutativity, we adopt a third interleavability side condition, which is a weaker, ordered, form of commutativity, defined as follows.

4.2 Definition (Interleavability). $p_{2}$ is interleavable after $p_{1}$, written $\operatorname{intrlv}\left(p_{1}, p_{2}\right)$ iff, for any interleaved evaluation of $p_{1}$ and $p_{2}$, that start from a given store, where the first call is to $p_{1}$.MoveNext: (a) the store result is the same; (b) the value result of $p_{1}$ is the same; (c) the value result of $p_{2}$ is the same.

While this sounds esoteric, $\operatorname{intrlv}\left(p_{1}, p_{2}\right)$ is a common condition, and is significantly weaker than $\operatorname{comm}\left(p_{1}, p_{2}\right)$. For example, it holds whenever $p_{1}$ is an eager plan, i.e., if it only accesses the shared store during its first step. Static approximation of intrlv $\left(p_{1}, p_{2}\right)$ is performed by extending path analysis so that it analyzes, for each physical path, what is accessed and updated by the whole plan, $a(p)$ and $u(p)$, and what is accessed an updated from the second step onwards $a_{2}(p)$ and $u_{2}(p)$. $\operatorname{intrlv}\left(p_{1}, p_{2}\right)$ is deduced when $a\left(p_{1}\right)$ and $u\left(p_{1}\right)$ do not interfere with $a_{2}\left(p_{2}\right)$ and $u_{2}\left(p_{2}\right)$. Both $a_{2}$ and $u_{2}$ are empty for eager plans, even if they are impure, which makes this condition widely applicable.

We now observe that to rewrite Join Hask $_{\mathrm{SEE}}$ into Join $\mathrm{H}_{\mathrm{SLE}}^{\mathrm{Hash}}$, we do not need $\operatorname{comm}\left(p_{3}, s_{1}\right)$, but $\operatorname{intrlv}\left(p_{3}, s_{1}\right)$ is enough, since both plans start executing $p_{3}$ before $s_{1}$. However, since the first steps of $p_{2}$ and $s_{1}$ are exchanged, $\operatorname{comm}\left(p_{2}, s_{1}\right)$ cannot be relaxed to $\operatorname{intrlv}\left(p_{2}, s_{1}\right)$.

We have proceeded in this way to specify the semantics of all our operators, and deduced the side conditions for the rewrite rules that we exploit to do code selection and to introduce pipelining; these are the rules in Figure 4. (Remark: Join Sort is a join implementation that can be used when $p_{1}$ is an order relation. It materializes $p_{3}$ and sorts it, and then iterates over $p_{2}$ and joins its result with the tuples from $p_{3}$ which are now in a convenient order.)

\section{EXPERIMENTS}

We implemented our approach using a publicly available XQuery processor [26], which we extended to support the core of the XQueryP language (without limitations on where side effects may occur). We also implemented side-effect analysis and modified the rewriting rules in the optimizer as described in this paper. We report on two classes of experiments, the first of which verifies the completeness and correctness of the compiler, while the second one looks at its performance.

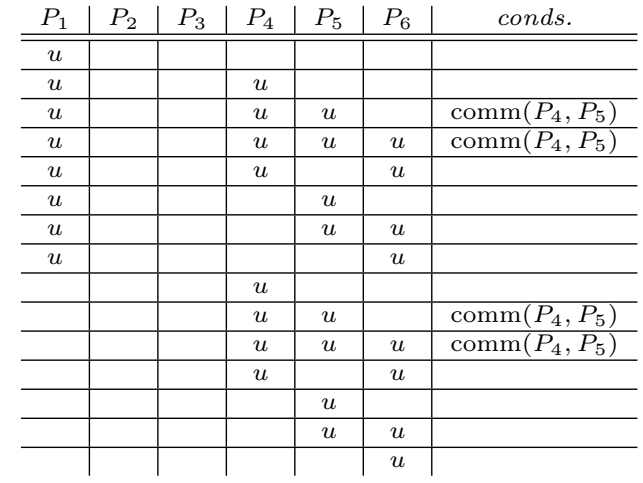

Table 1: Unnesting Study.

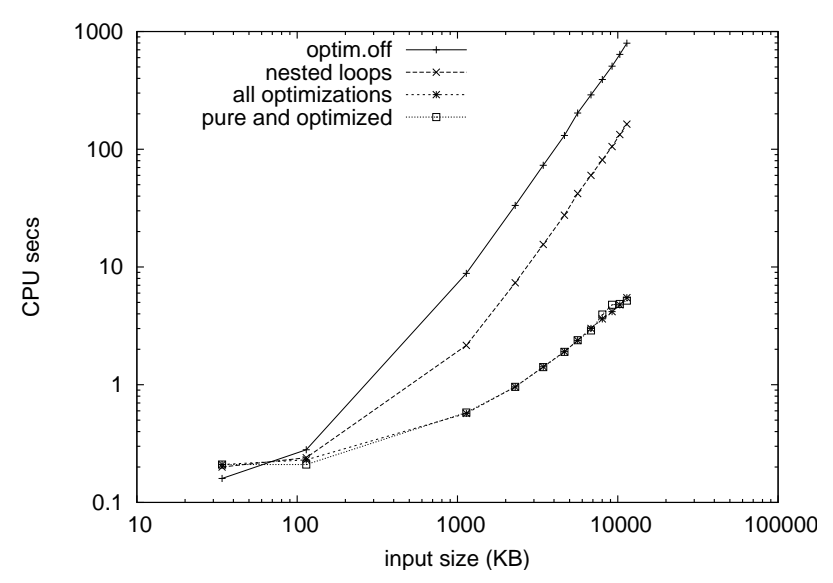

Figure 5: Running times for query q08.

\subsection{Completeness and Correctness}

We designed queries on the XMark [22] schema following the generic pattern of Scenario 3.5, and inserted updates into expressions $E_{1}, E_{4}, E_{5}, E_{6}$, such that the unnesting conditions are satisfied. The tests cover all possibilities of using updates in one, two, three or four of the expressions mentioned above. $E_{3}$ and $F_{2}$ did not contain update statements because $E_{3}$ always needs to be pure, ${ }^{1}$ and into $F_{2}$ we could not insert updates because of limitations of the GroupBy implementation in the original compiler. Table 1 summarizes all cases in which unnesting can be performed, depending on conditions satisfied by plans $P_{i}$ of each $E_{i}$. Each row characterizes one class of queries: the $u$ annotation in column $P_{i}$ indicates that $P_{i}$ contains update statements and the conds column describes additional conditions to be checked. We mention that all these queries could be pipelined, i.e., the output of the Map operators could be streamed and the left branch of the joins was evaluated lazily.

We ran the queries over a sample 1MB XMark document, checking that: (i) the optimized query plan is an instantiation of the generic optimized plan and (ii) the results obtained with and without optimizations are identical. We also tested queries covering all possibilities of adding updates inside the $E_{i}$ expressions such that the unnesting conditions were not satisfied. These are representatives of the

\footnotetext{
${ }^{1}$ The current implementation checks purity instead of the more general idempotency.
} 


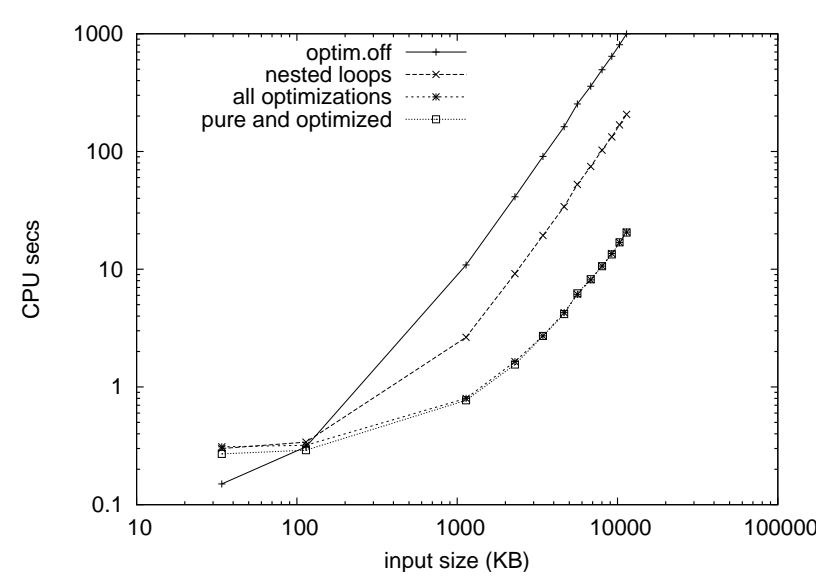

Figure 6: Running times for query q09.

classes of queries from Table 1 for which the additional conditions are violated, together with all other combinations of $u$ annotations not appearing in Table 1 (the cases in which $E_{3}$ contains updates). For them, we first checked that the optimizer does not push the MapConcat through GroupBy or that it does not introduce an outer join, depending on the condition that was violated. We verified that the evaluation of the partially optimized plans obtained for these queries produces the same results as in the case when optimizations are switched off. It is worth pointing out that both these tests were instrumental in finding several bugs in the implementation of the physical join operators and of the pipelining optimizations.

\subsection{Performances}

In order to validate the performance gains made possible by our optimizations, we tested XMark queries 8 to 12 , which perform various types of joins and grouping. We modified these queries by adding updates in the inner return clauses, corresponding to $E_{5}$ from our general unnesting form. The updates consist in modifying the statistics of the number of transactions, considering each inner for-loop to correspond to a transaction. The experiments were run on a machine with a Pentium $4 \mathrm{CPU}, 3.2 \mathrm{GHz}, 1 \mathrm{~GB}$ of RAM, running the etch distribution of Debian GNU/Linux.

We ran these queries on documents up to $11 \mathrm{MB}$ in three different scenarios: with all optimizations turned off, with optimizations on, but joins executed as nested loops, and finally with joins executed efficiently using hash or sort-merge join algorithms. Since we rely on an existing compiler, our goal is essentially to demonstrate that we could recover the proper behavior when side effects are present. As a point of reference, we also provide the running times obtained with the same queries after removing all update statements (which we call pure queries) and enabling all optimizations.

Figure 5 shows the running times (on a log-log scale) for query q08, which is an instance of the generic Scenario 3.5 performing an equi-(outer)join. As one can see, with optimizations on, the pure and the impure version behave almost the same. Also, as expected, executing joins as nested loops reduces the running time, but does not change the asymptotic behavior. Similar results were obtained for query q10 (See Figure 7).

The results for query q09 are shown on Figure 6. This

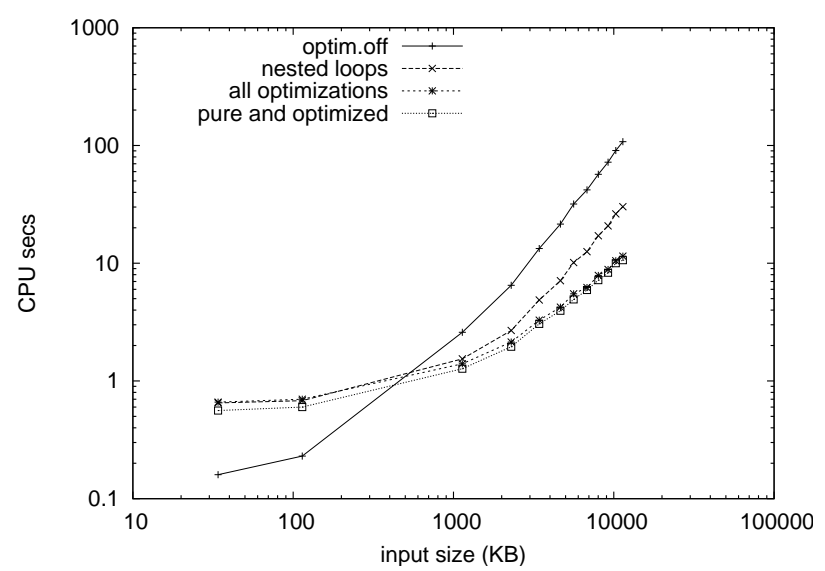

Figure 7: Running times for query q10.

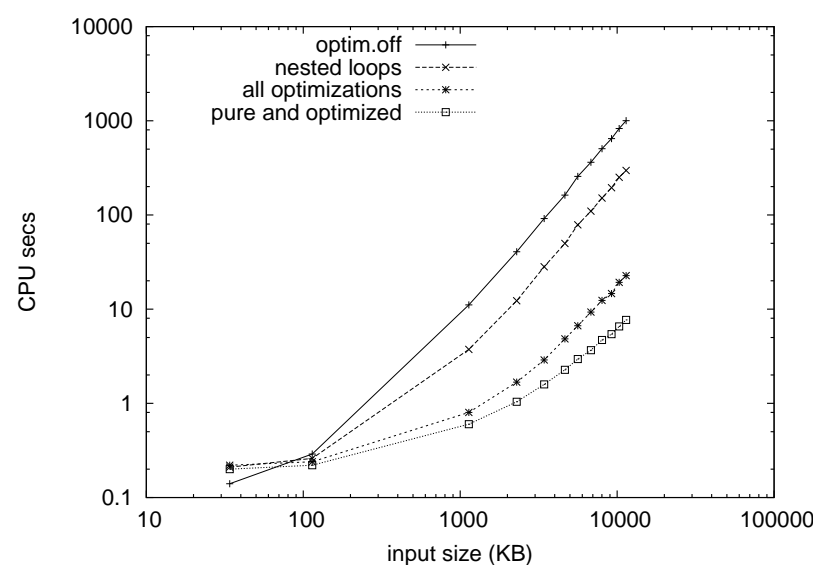

Figure 8: Running times for query q11.

query is a nested 3-way join and even though optimization leads to a substantial improvement in running time, it does not change the asymptotic behavior very significantly. Still, we can see that the optimized query with updates does behave similarly to the one without updates, which seems to point to a limitation in the original compiler.

Queries q11 and q12 are very similar, which is why we only show the graph for q11, in Figure 8. These are queries that touch a larger set of data, using a greater-than predicate in the join condition. Turning all optimizations on, which includes selecting a sort-merge physical join operator, leads to substantial gains: for instance, for the 11MB document, running query q11 takes $21 \mathrm{~s}$, compared to $998 \mathrm{~s}$ with the naive plan and q12 takes 22s, compared to $1,000 \mathrm{~s}$. Part of this gain is due to pipelining: if all intermediate results are materialized, evaluation of the optimized logical plans each take 44s. There is however a more noticeable increase in evaluation time than in q08-q10, explainable by the much larger number of updates that are performed (up to 118,271 updates compared to at most 3742 updates for q08-q10).

To provide a better image of how pipelining contributes to the overall optimization process, we display in Table 2 the evaluation times for $\mathrm{q} 12$ on a subset of the documents we used. As we can see, join optimizations alone (third column in Table 2), including use of the proper join al- 


\begin{tabular}{r|r|r|r} 
doc.size & no optim. & joins, but no pipelining & all optim. \\
\hline \hline $34 \mathrm{~KB}$ & $0.13 \mathrm{~s}$ & $0.23 \mathrm{~s}$ & $0.22 \mathrm{~s}$ \\
$1135 \mathrm{~KB}$ & $11.05 \mathrm{~s}$ & $1.25 \mathrm{~s}$ & $0.79 \mathrm{~s}$ \\
$3425 \mathrm{~KB}$ & $90.85 \mathrm{~s}$ & $5.27 \mathrm{~s}$ & $2.82 \mathrm{~s}$ \\
$5616 \mathrm{~KB}$ & $255.12 \mathrm{~s}$ & $13.01 \mathrm{~s}$ & $6.42 \mathrm{~s}$ \\
$8003 \mathrm{~KB}$ & $495.03 \mathrm{~s}$ & $24.56 \mathrm{~s}$ & $11.84 \mathrm{~s}$ \\
$11397 \mathrm{~KB}$ & $1000.36 \mathrm{~s}$ & $44.90 \mathrm{~s}$ & $21.52 \mathrm{~s}$
\end{tabular}

Table 2: Comparative running times for q12.

gorithm, reduces running time by one order of magnitude and adding pipelining (fourth column) provides an additional improvement with a factor between 1.5 and 2.1. Similar results were obtained for the other queries, namely a speedup factor between 1.32 and 3.43 (except for the $34 \mathrm{~KB}$ and $114 \mathrm{~KB}$ documents). The effect of pipelining is even more important-between 2.54 and 4.81 speedup for the non-optimized case - if we run these queries with pipelining turned on, but logical optimizations turned off. The explanation is that MapConcat and Select operators are used instead of Join and GroupBy, and require a significant amount of materialization. This seems to indicate that pipelining could be very important for fragment of the plans that cannot be optimized (e.g., when some side conditions do not hold).

\section{RELATED WORK}

There has been almost no work on optimization for query languages in the presence of side effects. The closest related work to ours is [13], considering join optimization for the XQuery! language. However, it does not provide a detailed analysis of the side conditions needed during rewriting. In the functional programming community, state monads are commonly used to support imperative features in pure languages $[20]$. A generally accepted opinion is that lazy evaluation and side effects are, from a practical point of view, incompatible [16]. This is consistent with our approach in which we use an eager semantics, and only introduce limited lazyness for the purpose of pipelining. Monads have been also been used to support query languages semantics and implementation $[3,9,24]$. However, this work does not consider side effects. The only exception seems to be Fegaras [10], who studies optimization for an object-oriented query languages with updates, by using a state monad but do not address algebraic optimization.

\section{CONCLUSION}

In this paper, we have presented a study of the impact of adding side-effects into an algebraic XML query compiler. We have shown that with the proper care and sufficient static analysis, one can safely extend a traditional database compiler with side-effecting operations. We believe this is an important step toward supporting a new generation of languages which blend database querying and imperative programming, such as XQuery scripting extensions. There is still much to do as future work, most notably in broadening the scope of rewritings being considered, and in the area of cost-based optimization.

\section{REFERENCES}

[1] BEA AquaLogic DSP documentation: Sample retail application

http://edocs.bea.com/aldsp/docs25/install/sampleapp.html.
[2] Scott Boag, Don Chamberlain, Mary F. Fernández, Daniela Florescu, Jonathan Robie, and Jérôme Siméon. XQuery 1.0: An XML Query Language, W3C Recommendation, 2007.

[3] Peter Buneman, Leonid Libkin, Dan Suciu, Val Tannen, and Limsoon Wong. Comprehension Syntax. SIGMOD Record, 23(1):87-96, 1994.

[4] Michael Carey. Data delivery in a service-oriented world: the BEA AquaLogic Data Services Platform. In SIGMOD Conference, pages 695-705, 2006.

[5] Don Chamberlain, Michael Carey, Daniela Florescu, Donald Kossmann, and Jonathan Robie. XQueryP: Programming with XQuery. In XIME-P, 2006.

[6] Don Chamberlain, Daniela Florescu, and Jonathan Robie. XQuery Update Facility, W3C Working Draft 11 July 2006, 2007.

[7] James Clark. XSL Transformations (XSLT), W3C Recommendation, 1999.

[8] Sophie Cluet. Designing OQL: Allowing Objects to be Queried. Information Systems, 23(5):279-305, 1998.

[9] Leonidas Fegaras. Query Unnesting in Object-Oriented Databases. In SIGMOD Conference, pages 49-60, 1998.

[10] Leonidas Fegaras. Optimizing Queries with Object Updates. J. Intell. Inf. Syst., 12(2-3):219-242, 1999.

[11] Steven Feuerstein and Bill Pribyl. Oracle $P L / S Q L$ Programming. O'Reilly, 2005.

[12] Daniela Florescu, Andreas Grünhagen, and Donald Kossmann. XL: An XML programming language for Web service specification and composition. In International conference on World Wide Web, pages 65-76, May 2002.

[13] Giorgio Ghelli, Christopher Re, and Jérôme Siméon. XQuery!: An XML Query Language with Side Effects. In EDBT Workshops, pages 178-191, 2006.

[14] Giorgio Ghelli, Kristoffer Høgsbro Rose, and Jérôme Siméon. Commutativity Analysis in XML Update Languages. In ICDT, pages 374-388, 2007.

[15] Jan Hidders, Jan Paredaens, and Roel Vercammen. On the Expressive Power of XQuery-Based Update Languages. In XSym, pages 92-106, 2006.

[16] Simon Peyton Jones. Tackling the awkward squad: monadic input/output, concurrency, exceptions, and foreign-language calls in Haskell. In "Engineering theories of software construction", ed Tony Hoare, Manfred Broy, Ralf Steinbruggen, IOS Press, 2001.

[17] The LINQ Project. http://msdn.microsoft.com/XML/linqproject.

[18] N. May, S. Helmer, and G. Moerkotte. Strategies for Query Unnesting in XML Databases. ACM Transactions on Database Systems, 31(3):968-1013, 2006.

[19] Guido Moerkotte. Building Query Compilers, Draft Manuscript. http://db.informatik.uni-mannheim.de/ moer, December 2005.

[20] Simon Peyton Jones and Philip Wadler. Imperative functional programming. In 20th Symposium on Principles of Programming Languages, North Carolina, USA, January 1993. ACM Press.

[21] Christopher Re, Jérôme Siméon, and Mary F. Fernández. A Complete and Efficient Algebraic Compiler for XQuery. In ICDE, page 14, 2006.

[22] Albrecht Schmidt, Florian Waas, Martin L. Kersten, Michael J. Carey, Ioana Manolescu, and Ralph Busse. XMark: A Benchmark for XML Data Management. In $V L D B$, pages 974-985, 2002.

[23] Philip Wadler. Web Development without Tiers. In 5th International Symposium on Formal Methods for Components and Objects, 2006.

[24] Limsoon Wong. Kleisli, a functional query system. Journal of Functional Programming, 10(1):19-56, 2000

[25] Details omitted due to double-blind reviewing.

[26] Details omitted due to double-blind reviewing. 